\title{
PATENT DUCTUS ARTERIOSUS WITH AN ABNORMAL AORTIC VALVE
}

In July, 1833, Dr. R. L. Nixon of Dublin was consulted by Dr. B., an English " medical gentleman," aged 27. "He was labouring under dyspepsia of rather an aggravated form, the result as he supposed of some alteration he had made during the preceding month in his usually very temperate habits." The aorta exhibited a " bruit de soufflet" through its whole course and beneath the anterior border of the liver was a pulsating mass. Dr. William Stokes was called and it was considered "that inflammation of the lining membrane of the aorta existed." In April, 1834, the patient died and Dr. Nixon demonstrated at necropsy that there was an aortic coarctation and a patent ductus arteriosus, but more remarkably, the aortic valve was " completely obliterated by an irregular fleshy mass. Several particles of phosphate of lime were deposited in the mass " (Nixon, 1834).

This is the first description we have discovered of aortic valve disease associated with a patent ductus arteriosus (P.D.A.). Hare (1859) described the post-mortem findings in a baby of six months, who had a bicuspid aortic valve and a P.D.A. Barie (1886) reported a patient with P.D.A., coarctation of the aorta, and aortic incompetence; in this case also the aortic valve lesion may have been a bicuspid valve. Morrison and Edwards (1950) described a patient who died when aged about twenty-six with a P.D.A. and a sub-aortic shelf in whom the aortic cusps were thickened and the commissures slightly fused. Gross and Longino (1951) made a clinical diagnosis of subaortic stenosis in three out of 412 patients operated on for P.D.A. and Burchell et al. (1953) add a further similar case among seven with P.D.A. and pulmonary hypertension. However, the certain clinical distinction between valvular and sub-aortic stenosis is impossible. Of 9 patients with congenital mitral stenosis reported by Ferencz et al. (1954) three had both aortic stenosis and a P.D.A. among other abnormalities.

\section{Clinical Material}

Eight cases are here reported of P.D.A. associated with aortic valve disease. They were found among about 190 cases of P.D.A. operated on at Great Ormond Street Hospital for Sick Children and St. Thomas' Hospital during the period 1947-53. This is a higher incidence than one would have expected in view of the sparse reference to this combination. The clinical and laboratory findings are therefore given in some detail.

That the P.D.A. was not the only abnormality present was evident in most of them before operation, but the exact nature of the complicating lesions was sometimes determined only after thoracotomy for ligation of the P.D.A. None of the patients have died. 


\section{CASE Summaries}

Case 1. G.P. (female). She remained well until the age of 17 months when she developed pneumonia and on examination by her own doctor was found to have an abnormal heart. At the age of 4 years she had subacute bacterial endocarditis following tooth extraction. Blood culture grew Streptococcus viridans and she was treated with penicillin and chloramphenicol at another hospital. Despite developing congestive cardiac failure she eventually made a good recovery.

A year later she was first seen at the Heart Clinic, Great Ormond Street. She was breathless on exertion. The præcordium bulged and the apex beat was abnormally forceful. There was marked arterial pulsation in the neck. A systolic thrill was felt on both sides of the sternum at the base, it was conducted up into the neck, and was accompanied by a systolic murmur with the same distribution. The blood pressure was $105 / 60 \mathrm{~mm}$. X-rays (Fig. 1) showed enlargement of the left ventricle, increased pulsation in the aorta,
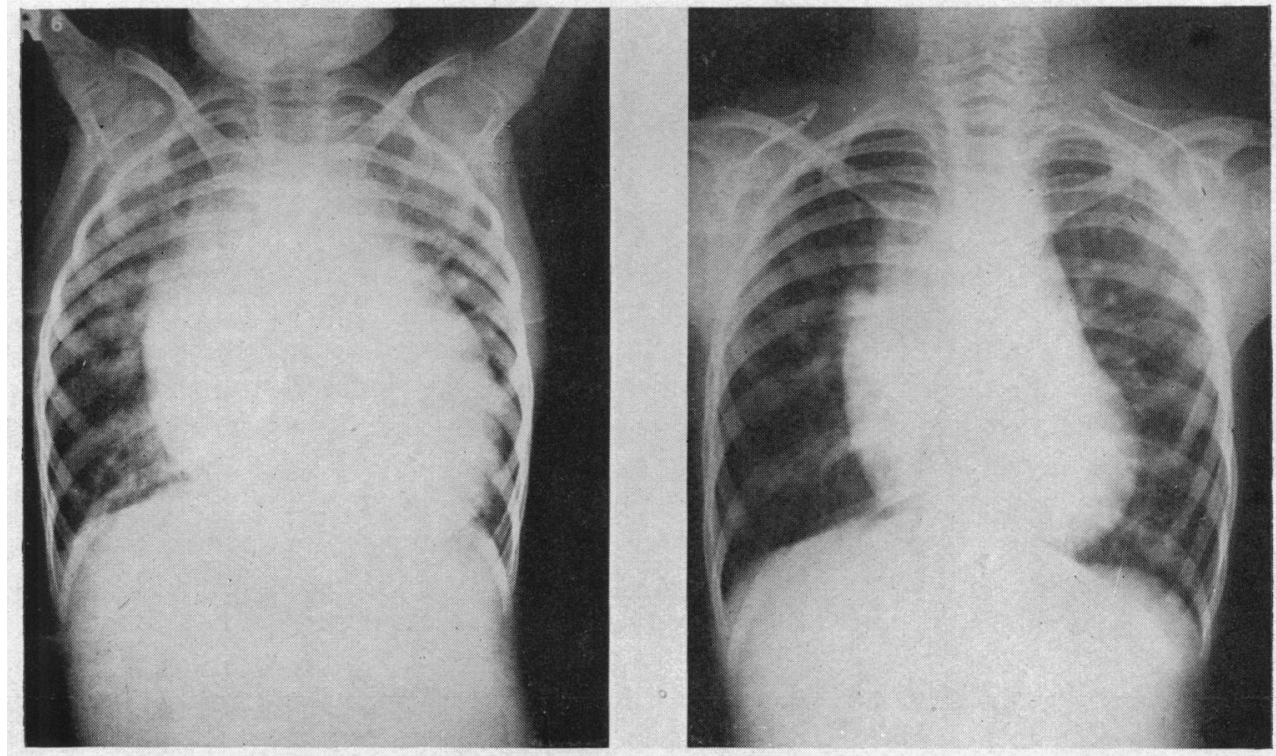

FIG. 1.-Case 1 before and after ligation of the patent ductus arteriosus, showing the decrease in heart size and pulmonary vascular markings after operation.

and increased pulmonary vascular markings. The electrocardiogram showed left ventricular hypertophy and on phonocardiography in the second left interspace the systolic murmur was seen to run up to the second sound which was followed by a short diastolic murmur. Six months later the murmurs in the pulmonary area had become continuous and at cardiac catheterization the catheter passed through a patent ductus arteriosus. At the age of six she was orerated on and a short aneurysmal ductus was ligated.

After operation a systolic thrill and murmur were still present in the suprasternal notch; they radiated up into the neck and were both also noted at the apex; there was also an early diastolic murmur. X-rays (Fig. 1) showed a reduction in heart size and there was less left ventricular hypertrophy cardiographically. A phonocardiogram one year after operation confirmed the presence of systolic murmurs at both the base and apex and the early diastolic murmur.

Summary. Patent ductus arteriosus, aortic stenosis and incompetence.

Case 2. G. M. (male). No abnormality was found until routine examination at the age of five disclosed a cardiac murmur. He did not play games at school-but gradually became able to walk quite long distances. He was admitted to Great Ormond Street Hospital when aged nine. He was underweight $(40 \mathrm{lb}$.), under height (48 in.), and anæmic. The apex beat was in the sixth interspace in the mid-axillary line. There was a systolic murmur and thrill maximal in the third left interspace and conducted up into the neck. A diastolic murmur was heard to the right of the sternum. The blood pressure was 110/40. X-rays showed gross cardiac enlargement mainly of the left ventricle and there was dilatation and increased pulsation of the aorta. The pulmonary vascular markings were increased. The cardiogram showed left ventricular hypertrophy 
and strain. At cardiac catheterization a left-sided superior vena cava was found and the catheter passed through a patent ductus arteriosus. Angiocardiography showed that there was also a right-sided superior vena cava. At the age of ten the ductus $(15 \mathrm{~mm}$. in diameter) was ligated.

After operation the pulse pressure remained high (130/40) and the cardiogram was unchanged. There was a systolic thrill and murmur and an early diastolic murmur in the second right interspace: these were demonstrated on phonocardiography. X-rays showed a slight decrease in the heart size and the pulmonary vascular markings returned to normal.

Summary. Patent ductus arteriosus, aortic stenosis and incompetence.

Case 3. R.Z. (male). At the eighth week of pregnancy, his mother had rubella. A cardiac murmur was heard at the age of six months. When aged 11 years he became short of breath on exertion and was first seen at Great Ormond Street Hospital. He had arachnodactyly and stippled retinæ. The præcordium bulged and the apex beat was in the anterior axillary line. There was a widespread præcordial systolic thrill conducted up into the neck, a loud systolic murmur in the third and fourth right intercostal spaces, and a continuous murmur in the pulmonary area. These murmurs were confirmed by phonocardiography, which showed the systolic murmur to be diamond shaped, and in addition a short diastolic murmur was seen at the apex. His blood pressure was 140/40. X-rays showed marked cardiac enlargement mainly of the left ventricle, and increased pulmonary vascular markings. The cardiogram showed left ventricular hypertrophy and strain. At cardiac catheterization the oxygen saturations were: right atrium and right ventricle 9.9 . vols. per $100 \mathrm{ml}$., right main branch of pulmonary artery 12.1 vols per $100 \mathrm{ml}$., but the catheter did not enter a patent ductus. At thoracotomy at the age of twelve a large ductus, $20 \mathrm{~mm}$. in diameter, entering the right main branch of the pulmonary artery, was ligated.

After operation his blood pressure was 90/50. There was a systolic thrill and systolic and early diastolic murmurs in the aortic area. X-rays showed a decrease in the heart size and the pulmonary vascular markings were now normal. The cardiogram remained unchanged.

Summary. Patent ductus arteriosus, aortic stenosis and incompetence.

Case 4. R.M. (male). He suffered from recurrent respiratory infections and epistaxes as an infant. At the age of two, a loud systolic murmur was heard. He was first seen at the Heart Clinic, Great Ormond Street, when seven, and had considerable dyspnœa on exertion. The apex beat was $3.7 \mathrm{~cm}$. outside the nipple line. There was a systolic thrill and murmur in the aortic area conducted up into the neck and a continuous thrill and murmur in the pulmonary area. There was also an early diastolic murmur down the left sternal edge. These murmurs were confirmed by phonocardiography (Fig. 2A). His blood pressure was $110 / 55$. X-rays showed enlargement of both ventricles, and enlargement of the pulmonary artery and its branches. The cardiogram indicated left ventricular hypertrophy. At cardiac catheterization the oxygen saturations in the right ventricle and pulmonary artery were 58 and 71 per cent respectively, but the catheter did not enter a ductus. At thoracotomy a vessel was ligated that was thought to be a ductus, but afterwards there was no change in the continuous murmur. It was thought that the left main branch of the pulmonary artery had probably been ligated in error and this conclusion was supported by finding much decreased vascular markings on the left side on screening. Four years later a further thoracotomy was done. The left main branch of the pulmonary artery was small and appeared to have no flow through it. A large ductus was found and ligated.

After operation he developed temporary congestive cardiac failure. On recovery his blood pressure was 110/80. There was a systolic thrill and murmur in the aortic area conducted into the neck and an early diastolic murmur down the left sternal edge (Fig. 2B). X-rays showed a decrease in the size and pulsation of the pulmonary vessels on the right side. The cardiogram showed no change.

Summary. Patent ductus arteriosus, aortic stenosis and incompetence.

Case 5. N.H. (male). His mother had rubella in the second month of pregnancy. He was known to have a heart lesion and bilateral cataracts at birth and after a few weeks was found to have bilateral deafness. During infancy he had recurrent chest infections. When first seen at St. Thomas' Hospital at the age of thirteen, he had slight dyspnœa on exertion and was undersized (weight $58 \mathrm{lb}$., height $57 \mathrm{in}$.). The left chest was prominent and the apex beat was in the anterior axillary line in the fifth intercostal space. There was a systolic thrill and murmur and also an early diastolic murmur in the second left intercostal space. There was marked pulsation in a prominent right common carotid artery. His blood pressure was 90/40. On radioscopy both ventricles appeared to be enlarged and the main pulmonary artery and its branches were increased in size and pulsation, and the aortic arch was high. The cardiogram appeared to show enlargement of both ventricles ( $T$ in aVL inverted, $R$ in V5 plus $S$ in V2 equals $50 \mathrm{~mm}$., $R$ in V4R equals $15 \mathrm{~mm}$.). At cardiac catheterization the pressures in $\mathrm{mm}$. Hg were: right atrium (R.A.) 4/1, right ventricle 
(R.V.) $108 / 0$, pulmonary artery (P.A.) $103 / 58$, aorta 120/70. Oxygen saturations were R.A. 68, R.V. 68, P.A. 79, and aorta 88 per cent. A left lateral angiogram showed a greatly enlarged innominate artery.

At thoracotomy at the age of fourteen, there was an aneurysmal dilatation of the arch of the aorta and the pulmonary artery was also very large. There was a very large patent ductus which was ligated. The P.A. pressure was $88 / 55$ before and $48 / 25 \mathrm{~mm}$. after ligation. Two weeks later his blood pressure was $100 / 70$ and there was a loud systolic murmur all over the præcordium maximal in the suprasternal notch and an early diastolic murmur down the left sternal edge. A phonocardiogram showed a diamond-shaped
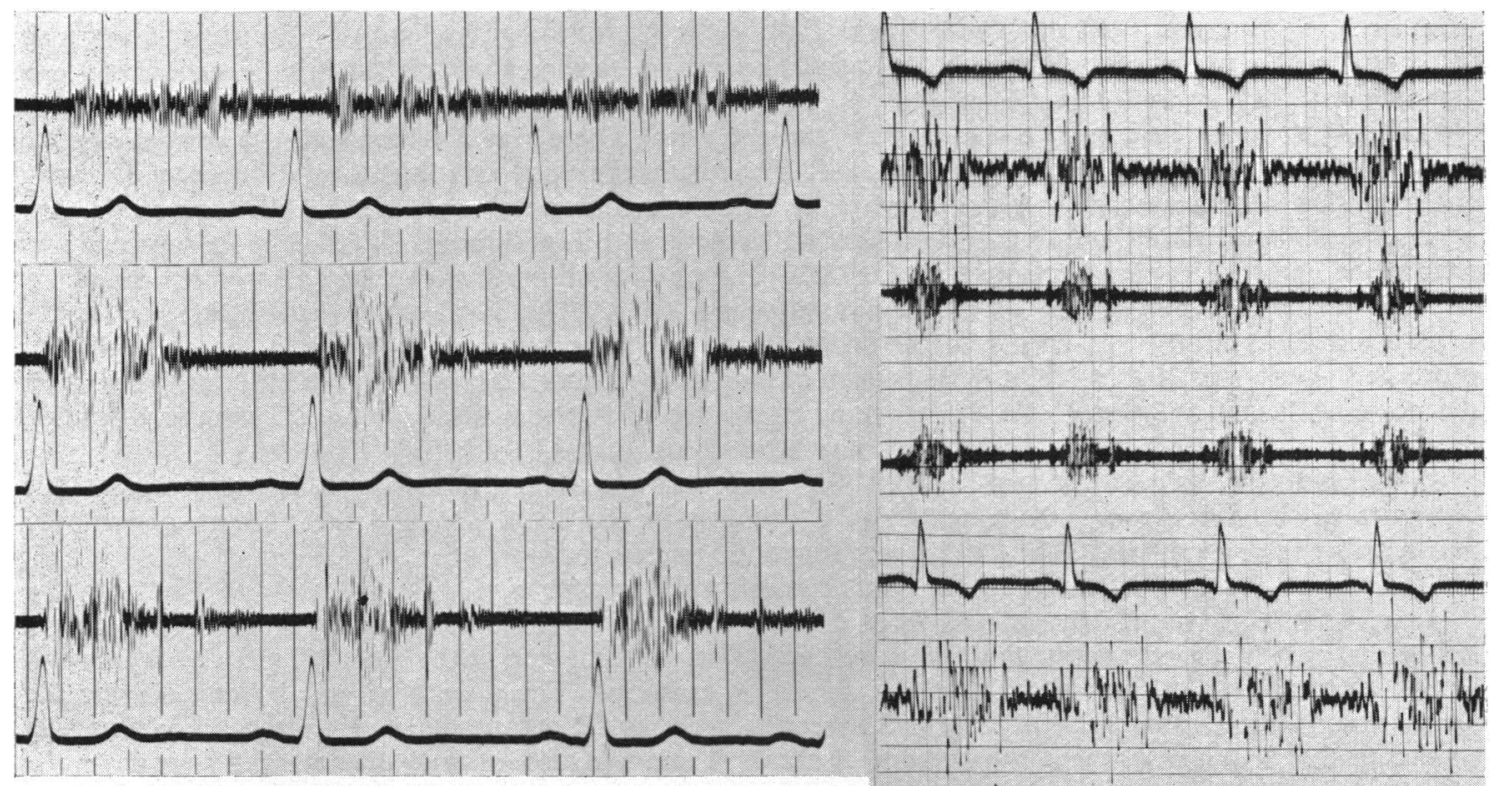

A

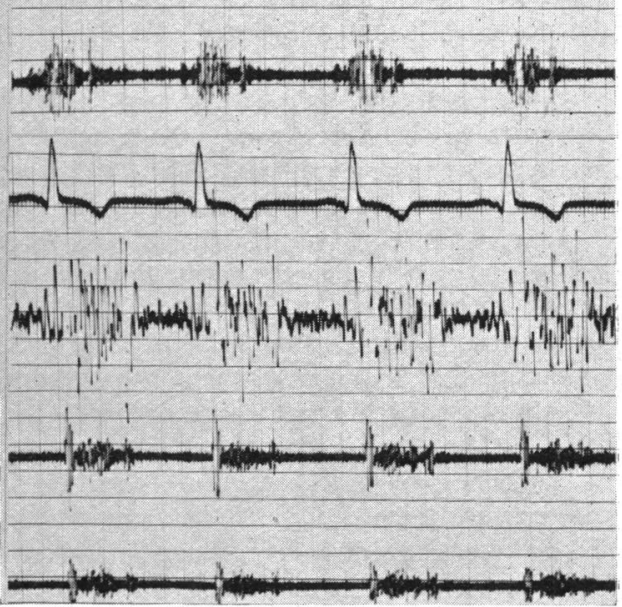

B

Fig. 2.-Case 4. (A) High frequency phonocardiograms before operation showing (from above downwards) a continuous murmur in the first left intercostal space, a systolic murmur of diamond shape, and an early diastolic murmur in the third left intercostal space, and a mid-diastolic murmur at the apex. (B) Phonocardiograms after operation. The upper three channels in the second right intercostal space (from above downwards: low, medium, and high frequencies) show a persistent diamond-shaped systolic murmur and an early diastolic murmur. The lower three channels in the second left intercostal space (from above downwards: low, medium, and high frequencies) show the disappearance of the continuous murmur.

systolic murmur at the base and apex and an early diastolic murmur at the base. A year later a chest $\mathrm{X}$-ray showed that the pulmonary artery and its branches were considerably smaller; the electrocardiogram had changed slightly, $\mathrm{T}$ in aVL now being isoelectric.

Summary. Patent ductus arteriosus, aortic stenosis and incompetence.

Case 6. A.A. (female). She was found to have a cardiac murmur when 10 days old. At the age of 9 years, she had subacute bacterial endocarditis (blood culture grew Streptococcus viridans) and after treatment with penicillin made an apparent complete recovery. When first seen at St. Thomas' Hospital a year later, she had slight dyspnœa on exertion. She was small for her age and thin. Her blood pressure was 130/0. There was unusually vigorous pulsation in the carotid, axillary, brachial; and retinal arteries and there was a pistol shot over all the peripheral arteries down to the radial and dorsalis pedis. There was a systolic and diastolic thrill and murmur maximal in the third left intercostal space. The murmurs appeared to be to-and-fro rather than continuous. X-rays showed left ventricular enlargement, a prominent first 
part of the aorta and a marked aortic fling. The main pulmonary artery was enlarged but its branches were normal. The cardiogram was normal. A phonocardiogram showed a systolic murmur maximal in mid systole in the second left intercostal space. There was no murmur at the second sound, which was followed by an early diastolic murmur. At cardiac catheterization the oxygen saturations were R.V. 66 and P.A. 76 per cent, and this suggested a patent ductus, although the clinical picture was dominated by severe aortic incompetence.

At thoracotomy a small patent ductus was ligated and the thrill previously palpable in the main pulmonary artery became less intense. Examination after operation showed no change in the signs. Two years later she was examined by Dr. Maurice Campbell and Dr. Paul Wood at the National Heart Hospital and they have kindly provided the results of a further catheterization: pressures, R.V. 57/0, P.A. 37/17 (indicating mild pulmonary stenosis); and oxygen saturations, P.A. and R.V. 76, R.A. 61, and brachial artery 94 per cent. This shunt was thought to indicate a ventricular septal defect but in view of the previous subacute bacterial endocarditis rupture of an aortic sinus into the right ventricle was a remote alternative. At the age of sixteen we found that the physical signs of gross aortic incompetence remained, her blood pressure being $220 / 0 \mathrm{~mm}$. The systolic thrill was still present in the fourth left intercostal space and may be caused by the ventricular septal defect. A cardiogram showed early left ventricular hypertrophy.

Summary. Patent ductus arteriosus, aortic incompetence, mild pulmonary stenosis, and probable ventricular septal defect. (Case E3, Table IX, Campbell, 1954).

Case 7. D.C. (female). At the age of seven she was found to have an abnormal heart. She was first seen at St. Thomas' Hospital at the age of fifteen and was then symptomless apart from slight dyspnœe on exertion. Her fingers showed arachnodactyly and she had a coloboma of the left iris. There was a lifting apex beat in the sixth intercostal space in the anterior axillary line. There was a continuous thrill maximal in the second left intercostal space with an extremely loud continuous murmur. To the right of the sternum there was an early diastolic murmur higher in pitch than the diastolic element of the continuous murmur. Her blood pressure was 115/35. X-rays (Fig. 3A) showed apparent enlargement of both ventricles, prominence of the first part and arch of the aorta, and enlargement of the main pulmonary artery and its

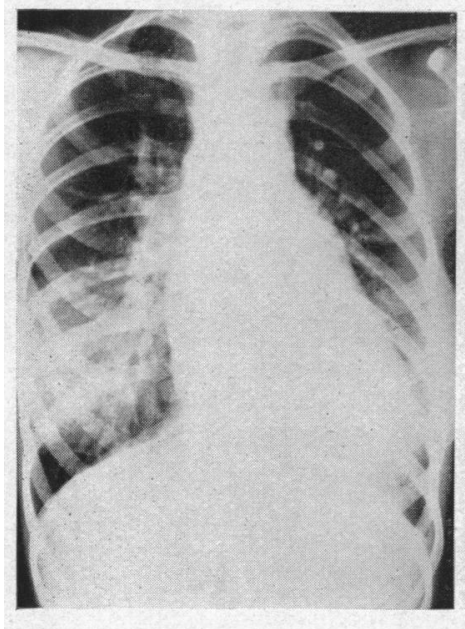

A

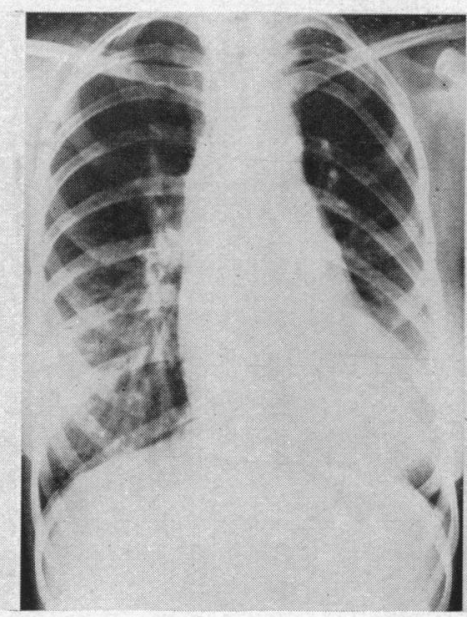

B

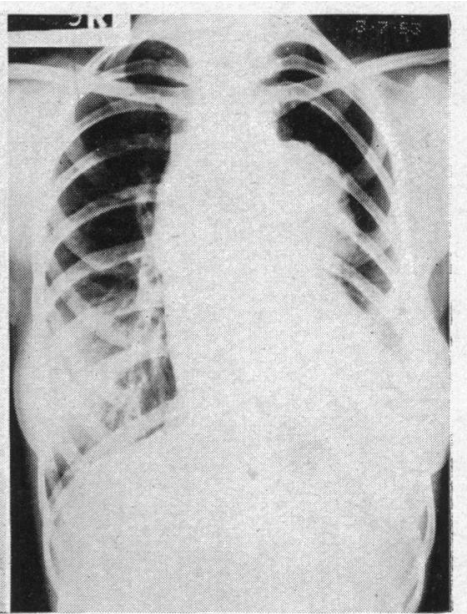

C

Fig. 3.-Case 7. (A) Before operation. (B) Four months, and (C) nine months after operation, showing decrease in heart size and pulmonary vascular markings in (B) and the aneurysmal dilatation of the pulmonary artery which later develped in (C).

branches. The cardiogram showed gross left ventricular strain. At cardiac catheterization the pressures in $\mathrm{mm}$. Hg were: R.A. mean 1 , R.V. 58/0, and P.A. 52/28, and the oxygen saturations were superior vena cava 56, inferior vena cava, 62, R.A. 57, R.V. 59, and left P.A. 80 per cent.

At thoracotomy a large patent ductus was found and ligated. There was slight dilatation of the aorta at its junction with the ductus and this became more marked after the ligation. After operation her blood pressure was 140/75 and there was a to-and-fro murmur to the right of the sternum but the continuous. 
murmur to the left of the sternum had gone. Two months later a quiet continuous murmur had returned and this was confirmed by phonocardiography (Dr. Leatham). However, the pulmonary artery and its branches were considerably smaller (Fig. 3B) and at cardiac catheterization the colour of the right ventricular and pulmonary arterial samples looked the same (due to a technical error they were not analysed), so it was concluded that the ductus had recanalized but that the shunt was only small. Nine months after operation she was found on routine chest X-ray to have developed an extreme aneurysmal dilatation of the pulmonary artery (Fig. 3C). Seventeen months after operation she felt very well and had started shorthand and typing. The signs of aortic incompetence and the X-ray appearances remained the same but the continuous murmur had disappeared.

Summary. Patent ductus arteriosus, aortic incompetence, possible recanalization of the ductus, development of aneurysm of pulmonary artery.

Case 8. R.R. (male). He was first seen at St. Thomas' Hospital when aged five. He was symptomless but a murmur had been heard by his doctor. His mother said that her sister, who lived with her, had had rubella when she, his mother, was two months pregnant. He had been born with a cataract in his right eye and despite an operation could count fingers only with this eye, and the visual acuity in his left eye was $6 / 36$; this was found by an ophthalmologist to be due to pigmentary degeneration at the macula. He was small for his age. There was a loud systolic murmur maximal in the pulmonary area but no diastolic murmur. A year later a diastolic murmur was heard and at the age of seven there was a continuous murmur in the second left intercostal space. It was noted that the systolic element of the murmur radiated both to the neck and to the back. At thoracotomy a ductus was found but there was also a coarctation of the aorta and because of that finding the ductus was not then ligated. On re-examination afterwards no circulatory abnormalities due to the coarctation were found; the femoral and radial pulses were synchronous and the blood pressure in the legs 115/65. At the age of ten cardiac catheterization showed: (pressures in $\mathrm{mm} . \mathrm{Hg}$ ) R.A. mean 0, R.V. 15/3, P.A. mean 13; and oxygen saturations, R.A. 60 , R.V. 60 , and P.A. 85 per cent. In view of the size of the shunt the ductus was ligated at a second operation. After operation there was a thrill and harsh systolic murmur at the base, equal both sides of the sternum but radiating up into the neck. There was no diastolic murmur. The cardiogram showed incomplete right bundle branch block.

Summary. Patent ductus arteriosus, aortic stenosis, and mild coarctation of the aorta.

\section{DisCUSSION}

All these patients were diagnosed before operation as having a patent ductus arteriosus (P.D.A.) either on the basis of the passage of a catheter through it or because of a shunt of arterialized blood into the pulmonary artery.

The frequency of associated anomalies and the presence of local signs suggesting aortic valve disease were the two chief features that distinguished them from uncomplicated cases of P.D.A.

Associated Anomalies. These will be considered under two headings, extracardiac and cardiac. The extracardiac anomalies were as follows. Four cases had congenital eye defects, stippled retinæ (Case 3), bilateral cataracts (Case 5), coloboma of the left iris (Case 7), and cataract in the right eye and pigmentary degeneration of the left macula (Case 8). Two of these (Cases 3 and 7) also had arachnodactyly and one (Case 5) had bilateral congenital deafness. Two of these (Cases 3 and 5) had a history of maternal rubella in the relevant pregnancy, and in one (Case 8) his mother was in contact with rubella while two months pregnant, but in the others pregnancy and delivery were normal. Four (Cases 2, 5, 6, and 8) were under weight.

The associated cardiovascular anomalies were as follows. Mild coarctation of the aorta was seen in one (Case 8): this was an operative finding and was not severe enough to produce arm hypertension, collateral vessels, or diminution or delay in the femoral pulses. One (Case 5) had a greatly enlarged innominate artery. In one (Case 6) cardiac catheterization two years after her P.D.A. had been ligated showed mild pulmonary stenosis and a probable ventricular septal defect. One (Case 7) developed an aneurysmal dilatation of the pulmonary artery after operation. Case 2 had a double superior vena cava.

Signs suggesting Aortic Valve Disease. These can be divided into signs produced at the abnormal valve itself and signs indicating increased left ventricular work.

In three of the four patients with a continuous murmur to the left of the sternum an aortic 
valve lesion was suspected before operation on the basis of a systolic thrill maximal to the right of the sternum (Case 4), phonocardiographic evidence that the systolic murmur was maximal in mid systole (Case 3), and a diastolic murmur to the right of the sternum differing in pitch from the diastolic element of the continuous murmur (Case 7). Of the four patients without a continuous murmur three also were suspected of having an aortic valve lesion preoperatively because two (Cases 1 and 2) had a systolic thrill conducted up into the neck, and the other (Case 6) had a very high pulse pressure and pistol shot sounds over the peripheral arteries suggesting aortic incompetence. In Cases 5 and 8, an aortic valve lesion was not diagnosed until after the abolition of the murmurs of the P.D.A.

The signs indicating increased left ventricular work were: cardiac enlargement with a left ventricular type of apex beat ( 7 cases) and left ventricular hypertrophy both on screening ( 7 cases) and in the electrocardiogram ( 5 cases). In the absence of aortic incompetence, if a case of P.D.A. has a comparatively small pulse pressure (indicating a moderate or small shunt) the presence of left ventricular hypertrophy suggests aortic stenosis.

Post Operative Course. Cases 1-4 were operated upon by Mr. D. J. Waterston at Great Ormond Street Hospital for Sick Children, and Cases 5-8 by Mr. N. R. Barrett at St. Thomas' Hospital.

In six patients the P.D.A. was large and in two (Cases 1 and 3) of these, the cardiothoracic ratio dropped from 75 and 70 to 59 and 55 per cent respectively after operation. In five of these patients the pulmonary vessels became much smaller after operation but in the remaining one (Case 7) after an initial decrease there developed the aneurysmal dilatation of the pulmonary artery previously described. In the two patients with a small P.D.A. (Cases 6 and 8) the X-ray appearances after operation were unchanged. In no case did the cardiographic changes of left ventricular hypertrophy return to normal after operation.

Aortic valvotomy has not been attempted in any of these patients, but in those with aortic stenosis in whom a large left ventricular load persists after ligation of the P.D.A. it might be considered in the future.

\section{SUMMARY}

Eight patients with a patent ductus arteriosus and an aortic valve lesion are presented and the reported cases are briefly reviewed.

An aortic valve lesion may be suggested either by murmurs and thrills recognizably separate from those of the patent ductus or by evidence of left ventricular hypertrophy out of proportion to the size of the shunt.

Associated cardiovascular and other anomalies were common in these cases. In two or perhaps in three the mother had suffered from rubella in the second month of her pregnancy.

We are grateful to Mr. D. J. Waterston and Mr. N. R. Barrett for their co-operation, to Dr. Maurice Campbell and Dr. Paul Wood for supplying the results of cardiac catheterization at the National Heart Hospital in Case 6, to Dr. B. Weeks, Dr. Aubrey Leatham, and Dr. E. D. H. Cowen for the phonozardiograms in Cases 4, 7, and 6 respectively, and to Messrs. D. Martin and K. G. Moreman for the photographs.

\section{REFERENCES}

Barie, E. (1886). Rev. de Med., 6, 409.

Burchell, H. B., Swan, H. J. C., and Wood, E. H. (1953). Circulation, 8, 681.

Campbell, M. (1954). Brit. Heart J., 16. 296.

Ferencz, C., Johnson, A. L., and Wiglesworth, F. W. (1954). Circulation, 9, 161.

Gross, R. E., and Longino, L. A. (1951). Circulation, 3, 125.

Hare (1859). Trans. Path. Soc. Lond., 11, 46.

Morrison, R. W., and Edwards, J. E. (1950). Bull. Internat. Ass. Med. Mus., 31, 73.

Nixon, R. L. (1834). Dublin J. Med. Chem. Soc., 5, 386. 